\title{
Esofagectomia trans-hiatal versus transtorácica: Experiência do Instituto Nacional do Câncer (INCA)
}

\section{Transhiatal versus transthoracic esophagectomy: Experience of the Brazilian National Cancer Institute}

Emerson luis Gama Prisco, AsCBC-RJ'; Carlos Eduardo Pinto, tCBC-RJ²; Aldo Vieira Barros, AsCBC-RJ';

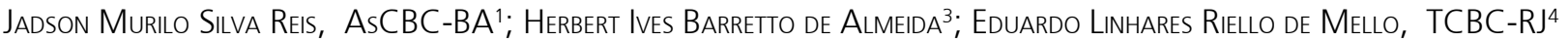

R E S U M O

\begin{abstract}
Objetivo: Analisar comparativamente a morbimortalidade e sobrevida após esofagectomia trans-hiatal (TH) ou transtorácica (TT). Metodos: Estudo retrospectivo não randomizado de 68 pacientes com neoplasia de esôfago operados no INCA entre 1997 e 2005 , divididos em dois grupos: 1 - TH (33 pacientes); e 2 - TT (35 pacientes). Resultados: A idade média foi 40,7 anos (25 - 74 anos), sendo $73,5 \%$ homens. Tumores do $1 / 3$ médio predominaram no Grupo $2(48,6 \%$ versus $21,2 \%, p=0,02)$. A média de linfonodos dissecados foi maior no Grupo 2 (21,6 versus 17,8 linfonodos, $p=0,04)$, porém sem diferença no número de linfonodos metastáticos $(4,1$ versus 3,9 linfonodos, $p=0,85$ ). O tempo cirúrgico médio foi maior no Grupo 2 (410 versus 270 minutos, $p=0,001$ ). 0 tempo médio de internação também foi maior no Grupo 2 (19 versus 14 dias, $p=0,001$ ). A morbidade operatória foi 50\%, sem diferença significativa $(42,4 \%$ versus $57,1 \%, p=0,23)$. Fístula esofágica ocorreu em $13,2 \%$, sem diferença significativa $(9,1 \%$ versus $17,1 \%$, $p=0,23$ ). A mortalidade foi 5,8\% (04 pacientes), sem diferença significativa $(1,4 \%$ versus $4,4 \%, p=0,83)$. Conclusão: Neste estudo, a morbimortalidade não apresentou diferença em relação à via de acesso para a esofagectomia, apesar do maior tempo cirúrgico e de permanência hospitalar na via TT. A sobrevida global em 3 e 5 anos também foi maior na TT, possivelmente devido a maior freqüência de estágios iniciais em pacientes submetidos à transtorácica.
\end{abstract}

Descritores: Neoplasias esofágicas. Esofagectomia. Morbidade. Mortalidade.

\section{INTRODUÇÃO}

O câncer de esôfago é considerado a terceira neoplasia mais comum do trato gastrointestinal e está entre as dez neoplasias mais prevalentes no mundo. Anualmente, ocorrem cerca de 400 mil novos caso de câncer de esôfago no mundo, especialmente em indivíduos do sexo masculino, tabagistas e com idade avançada'.

A incidência desta neoplasia apresenta grande variabilidade geográfica, sendo mais comum nos países da Ásia, principalmente na China e no Japão. Apresenta incidência elevada inclusive na África, América do Sul e em alguns países da Europa Ocidental (França e Suíça). Segundo o Ministério da Saúde do Brasil2 ${ }^{2}$, esta neoplasia tem expectativa de 10.550 novos casos em 2008, representando a $9^{a}$ maior incidência de câncer, com previsão de 7900 casos em homens. O adenocarcinoma tem uma incidência crescente a cada ano, devido principalmente a metaplasia intestinal do esôfago distal (Barrett) em conseqüência da doença do refluxo gastroesofágico (DRGE) .

O câncer de esôfago é uma enfermidade inicialmente pouco sintomática e com diagnóstico estabelecido em fases avançadas na maioria dos casos. Isso leva a elevada taxa de mortalidade e prognóstico pouco favorável ${ }^{3,4}$. Diagnóstico precoce e conduta terapêutica adequadas são imprescindíveis para o sucesso terapêutico. A prevalência de doença localmente avançada e metástase linfonodal é alta no momento do diagnóstico, o que compromete o tratamento e prognóstico destes pacientes ${ }^{1,2}$. A profundidade da invasão tumoral na parede do órgão e o status linfonodal são os fatores prognósticos mais importantes 5 .

Com o desenvolvimento de novas técnicas cirúrgicas e anestésicas e o avanço dos cuidados peri-operatórios, observou-se um aumento considerável na sobrevida dos pacientes submetidos à esofagectomia por câncer de esôfago. De modo inverso, houve diminuição da taxa de

Trabalho realizado na Seção de Cirurgia Abdomino-Pélvica do Instituto Nacional de Câncer (INCA) - Ministério da Saúde do Brasil - Rio de Janeiro - RJ- BR.

1. Cirurgião do Hospital Aristides Maltez - Liga Baiana Contra o Câncer- Salvador - BA-BR. 2. Coordenador do Grupo de Esôfago da Seção de Cirurgia Abdômino-Pélvica do INCA - Rio de Janeiro - RJ- BR; 3. Residente de Cirurgia Oncológica do INCA- Rio de Janeiro - RJ- BR; 4. Chefe do Departamento de Cirurgia Abdômino-Pélvica do INCA- Rio de Janeiro - RJ- BR. 
complicação pós-operatória, cirurgia com doença residual e comprometimento linfonodal. O aumento na sobrevida dos pacientes submetidos à esofagectomia por câncer de esôfago deveu-se especialmente à melhora nas técnicas anestésica e de cuidados peri-operatórios, que permitiram menores taxas e melhor controle das complicações pósoperatórias. Ao mesmo tempo, ocorreu um refinamento na técnica operatória, que levou a uma maior taxa de ressecções radicais, com controle de doença residual por comprometimento linfonodal.

A técnica empregada para o acesso cirúrgico em esofagectomias é importante fonte de estudos e os autores consideram equivalentes tanto a via trans-hiatal (TH) como a transtorácica (TT), entretanto, pondera-se os aspectos positivos e negativos de cada técnica ${ }^{6-8}$. A via TT com anastomose esofagogástrica intra-torácica foi a abordagem padrão desde a década de 1940, quando foi descrita por Ivor Lewis et al..$^{9}$ cursando com altas taxas de complicações respiratórias e mediastinite séptica associada a fístula anastomótica intra-torácica. Em 1976, McKeown ${ }^{10}$ descreveu uma técnica TT adicionando uma incisão cervical para anastomose cervical no tratamento de tumores do esôfago, principalmente dos terços superior e médio, diminuindo assim a taxa de complicações graves. Em 1978, Orringer e Sloan ${ }^{11}$ descreveram a técnica TH sem toracotomia e com anastomose cervical, evitando aquelas complicações temidas na via TT, cursando por vezes com uma benigna fístula salivar cervical em lugar da temida mediastinite séptica, cuja mortalidade atualmente está entre $20 \%$ e 35\% quando associada a presença de fístula intra-torácica ${ }^{12}$. As críticas à via TH baseiam-se na dissecção mediastinal sem visão direta, com comprometimento da hemostasia e incompleta dissecção linfonodal ${ }^{13}$.

O objetivo deste estudo é analisar comparativamente as vias TH e TT em relação a morbimortalidade operatória e a sobrevida global em pacientes submetidos à esofagectomia por neoplasia de esôfago.

\section{MÉTODOS}

Este é um estudo retrospectivo não randomizado baseado no banco de dados prospectivo e na revisão de prontuários da Seção de Cirurgia Abdômino-Pélvica (SCAP) do Instituto Nacional de Câncer (INCA). Um total de 68 pacientes com diagnóstico histológico de neoplasia de esôfago em terço médio ou inferior foram submetidos à esofagectomia pelas técnicas TH ou TT no período entre janeiro de 1997 e dezembro de 2005, distribuidos em dois grupos: Grupo 1 - operados por via TH, com 33 pacientes (48,5\%); e Grupo 2 - operados por via transtorácica, com 35 pacientes $(51,5 \%)$. Foram excluídos os pacientes que receberam tratamento neoadjuvante, esofagectomia de resgate ou que tiveram margem cirúrgica comprometida após a ressecção.
Todos os pacientes receberam estadiamento segundo as normas do TNM empregado pelo American Joint Committee on Cancer (AJCC - 2002) e foram selecionados primariamente para o tratamento cirúrgico os pacientes com estádio clinico I, IIA ou IIB, o performance status (PS) 0, 1 ou 2 pela escala de Zubrod (Eastern Clinical Oncology Group, ECOG), presença de doença ressecável na avaliação pré-operatória e condições clínicas adequadas para a realização do procedimento. A extensão inicial da doença foi avaliada rotineiramente pelo cirurgião oncológico através de exame clínico, endoscopia digestiva alta, tomografia computadorizada (TC) de tórax, abdome e pelve, radiografia de tórax, exame histopatológico (nova biópsia ou revisão por patologista do INCA) e laparotomia exploradora (ou laparoscopia). Eventualmente outros exames foram realizados (ressonância nuclear magnética, angio-TC, PET - positron emission tomography). As condições clínicas foram avaliadas por médico clinico da SCAP através de exames laboratoriais, eletrocardiograma, prova de função respiratória e outros, caso necessário. Os pacientes foram encaminhados à fisioterapia pré-operatoria e os tabagistas foram encaminhados ao Programa INCA Livre do Tabaco (PIL), além de suporte nutricional ou psicológico quando indicado.

Analisamos comparativamente os dois grupos em relação a morbimortalidade cirúrgica e sobrevida global. A mortalidade operatória foi considerada como óbito nos primeiros 30 dias de pós-operatório ou intra-hospitalar. A morbidade operatória foi considerada como qualquer seqüela clínica ou cirúrgica decorrente da operação, porém nós avaliamos principalmente as complicações precoces. O seguimento foi encerrado em dezembro de 2007.

Para comparação de proporções foi utilizado o teste não-paramétrico de qui-quadrado. O teste " $\mathrm{t}$ " de Student foi aplicado para comparação de médias aritméticas. Para comparação de valores que apresentam acentuada variação foi utilizado o teste não-paramétrico de Kruskal-Wallis. Para o cálculo da sobrevida global em cinco anos estimada em meses foi aplicado o método de Kaplan-Meier e para comparação do comportamento das curvas foi aplicado o teste não-paramétrico de Wilcoxon. Para validação estatística foi adotado o nível de significância de $5 \%$ de probabilidade $(p<0,05)$.

\section{RESULTADOS}

A análise demográfica está representada na tabela 1. A média de idade foi 40,7 anos (25 a 74 anos), sendo 50 homens $(73,5 \%)$ e 18 mulheres $(26,5 \%)$. A maioria dos pacientes tinham PS-0 ou 1 (82,4\%), sem diferença significativa entre os grupos $(p=0,09)$. Os grupos foram comparáveis para análise estatística.

Ao exame histopatológico (Tabela 2), a média de tamanho foi 5,05cm (1,3 a 15,0cm), sem diferença significativa entre os grupos. Predominaram os tumores do 
Tabela 1 - Características demográficas.

\begin{tabular}{|c|c|c|c|c|}
\hline & Total $(n=68)$ & Grupo $1(n=33)$ & Grupo $2(n=35)$ & $p$ \\
\hline Idade média (variação) & $55,4(25-74)$ & $55,4(25-72)$ & $55,4(35-74)$ & 1,00 \\
\hline \multicolumn{5}{|l|}{ Gênero } \\
\hline Masculino & $49 \quad(73,5 \%)$ & $25 \quad(75,8 \%)$ & $24 \quad(68,6 \%)$ & 0,52 \\
\hline Feminino & $18 \quad(26,5 \%)$ & $08 \quad(24,2 \%)$ & $11 \quad(31,4 \%)$ & \\
\hline \multicolumn{5}{|l|}{ OS } \\
\hline 0 & $12 \quad(17,6 \%)$ & $08 \quad(24,2 \%)$ & $04 \quad(11,4 \%)$ & 0,09 \\
\hline 1 & $45 \quad(66,2 \%)$ & $19 \quad(57,6 \%)$ & $26 \quad(74,3 \%)$ & \\
\hline 2 & $11 \quad(16,2 \%)$ & $06 \quad(18,2 \%)$ & $05 \quad(14,3 \%)$ & \\
\hline
\end{tabular}

Tabela 2 - Características histopatológicas.

\begin{tabular}{|c|c|c|c|c|c|c|c|}
\hline & \multicolumn{2}{|c|}{ Total $(n=68)$} & \multicolumn{2}{|c|}{ Grupo $1(n=33)$} & \multicolumn{2}{|c|}{ Grupo $2(n=35)$} & $p$ \\
\hline \multicolumn{8}{|l|}{ Tamanho (cm) } \\
\hline Média & \multicolumn{2}{|c|}{5,05} & \multicolumn{2}{|c|}{5,06} & \multicolumn{2}{|c|}{5,02} & 0,91 \\
\hline Variação & \multicolumn{2}{|c|}{$1,3-15$} & \multicolumn{2}{|c|}{$1,5-15$} & \multicolumn{2}{|c|}{$1,3-10$} & - \\
\hline \multicolumn{8}{|l|}{ Topografia } \\
\hline Esôfago médio & 24 & $(35,3 \%)$ & 07 & $(21,2 \%)$ & 17 & $(48,6 \%)$ & 0,02 \\
\hline Esôfago inferior & 44 & $(64,7 \%)$ & 26 & $(78,8 \%)$ & 18 & $(51,4 \%)$ & \\
\hline \multicolumn{8}{|l|}{ Tipo histológico } \\
\hline Adenocarcinoma & & $(47 \%)$ & 18 & $(54,6 \%)$ & 14 & $(40 \%)$ & 0,22 \\
\hline Escamoso & 34 & $(50 \%)$ & 14 & $(42,4 \%)$ & 20 & $(57,1 \%)$ & \\
\hline Leiomioma & 01 & $(1,5 \%)$ & 01 & $(3 \%)$ & - & & - \\
\hline Neuro-endócrino & 01 & $(1,5 \%)$ & - & & 01 & $(2,9 \%)$ & - \\
\hline \multicolumn{8}{|l|}{ Grau de diferenciação } \\
\hline I & 03 & $(4,4 \%)$ & 01 & $(3 \%)$ & 02 & $(5,7 \%)$ & 0,89 \\
\hline$\|$ & 51 & $(75 \%)$ & 25 & $(75,8 \%)$ & 26 & $(74,3 \%)$ & \\
\hline III & 11 & $(16,1 \%)$ & 05 & $(15,2 \%)$ & 06 & $(17,1 \%)$ & \\
\hline Indiferenciado & 01 & $(1,5 \%)$ & - & & 01 & $(2,9 \%)$ & - \\
\hline Boderline & 01 & $(1,5 \%)$ & 01 & $(3 \%)$ & - & & - \\
\hline in situ & 01 & $(1,5 \%)$ & 01 & $(3 \%)$ & - & & - \\
\hline \multicolumn{8}{|l|}{ Linfonodos dissecados } \\
\hline Média & 19 & & $17, \varepsilon$ & & 21,6 & & 0,04 \\
\hline Variação & & 51 & & 51 & & -33 & - \\
\hline \multicolumn{8}{|c|}{ Linfonodos metastáticos } \\
\hline Média & 04 & & 3, & & 4,1 & & 0,85 \\
\hline Variação & & 26 & 0 & 26 & & -20 & - \\
\hline
\end{tabular}

terço inferior no total, com 44 casos (64.7\%), principalmente no Grupo 1 (78,8\% versus 51,4\%, $p=0,02$ ), porém o Grupo 2 teve mais que o dobro de tumores do terço médio (48,6\% versus $21,2 \%, p=0,02)$. Grupo 1 teve maior incidência de adenocarcinoma, porém sem significância estatística (54,6\% versus $40 \%, p=0,22)$. Houve ainda um caso de carcinoma epidermóide in situ (1,5\%), um de leiomioma borderline no Grupo 1 (3\%) e outro de carcinoma neuroendócrino indiferenciado tipo pequenas células no Grupo 2 (2,9\%). O grau histológico II (moderadamente diferenciado) foi o mais comum (75\%), sem diferença significativa entre os grupos $(p=0,89)$. A média de linfonodos dissecados foi 19,1 (2 - 51 linfonodos), sendo significativa- mente maior no Grupo 2 (21,6 versus 17,8 linfonodos, $p=$ $0,04)$, porém não houve diferença significativa no número de linfonodos metastáticos (4,1 versus 3,9 linfonodos, $p=$ $0,85)$. As margens cirúrgicas foram livres de neoplasia em todos os casos.

O tempo cirúrgico médio foi 330 minutos $(180$ a 580 minutos) sendo maior no Grupo 2 (270 versus 410 minutos, $p=0,001)$. A média do tempo de internação foi 15 dias (5 a 90 dias) e também foi significativamente maior no Grupo 2 (14 versus 19 dias, $p=0,001$ ).

A morbidade operatória foi $50 \%$, sem diferença significativa entre os grupos $(42,4 \%$ versus $57,1 \%, p=$ 0,23), havendo 17 pacientes (25\%) com complicações ci- 
Tabela 3 - Morbimortalidade operatória.

\begin{tabular}{|c|c|c|c|c|c|c|c|}
\hline \multirow[b]{2}{*}{ Morbidade operatória } & \multicolumn{2}{|c|}{ Total $(n=68)$} & \multicolumn{2}{|c|}{ Grupo $1(n=33)$} & \multicolumn{2}{|c|}{ Grupo $2(n=35)$} & \multirow{2}{*}{$\begin{array}{c}p \\
0,23\end{array}$} \\
\hline & 34 & $(50 \%)$ & 14 & $(42,4 \%)$ & 20 & $(57,1 \%)$ & \\
\hline Cirúrgica & 17 & $(25 \%)$ & 07 & $(21,2 \%)$ & 10 & $(28,5 \%)$ & 0,41 \\
\hline Fístula cervical & 09 & $(13,2 \%)$ & 03 & $(9,1 \%)$ & 06 & $(17,1 \%)$ & 0,23 \\
\hline Necrose do tubo gástrico & 03 & $(4,4 \%)$ & 01 & $(3 \%)$ & 02 & $(5,7 \%)$ & 0,71 \\
\hline Fistula pancreática & 01 & $(1,5 \%)$ & - & & 01 & $(2,8 \%)$ & - \\
\hline Hemorragia da ABD & 01 & $(1,5 \%)$ & 01 & $(3 \%)$ & - & & - \\
\hline Hemorragia da VJIE & 01 & $(1,5 \%)$ & - & & 01 & $(2,8 \%)$ & - \\
\hline Brida & 01 & $(1,5 \%)$ & 01 & $(3 \%)$ & - & & - \\
\hline Infecção de sitio cirúrgico & 01 & $(1,5 \%)$ & 01 & $(3 \%)$ & - & & - \\
\hline Empiema pleural & 01 & $(1,5 \%)$ & - & & 01 & $(2,8 \%)$ & - \\
\hline Clínica & 19 & $(27,9 \%)$ & 10 & $(30,3 \%)$ & 09 & $(25,7 \%)$ & 0,67 \\
\hline Pneumonia & 18 & $(26,5 \%)$ & 09 & $(27,3 \%)$ & 09 & $(25,7 \%)$ & 0,67 \\
\hline Infecção do trato urinário & 01 & $(1,5 \%)$ & 01 & $(3 \%)$ & - & - & \\
\hline \multicolumn{8}{|l|}{ Reconstrução } \\
\hline Tubo Gástrico & 58 & $(85,3 \%)$ & 32 & $(97 \%)$ & 26 & $(74,3 \%)$ & 0,01 \\
\hline Cólon & 10 & $(14,7 \%)$ & 01 & $(3 \%)$ & 09 & $(25,7 \%)$ & 0,001 \\
\hline Tempo operatório (minutos) & 330 & $(180-580)$ & 270 & $(180-360)$ & 410 & $(240-580)$ & 0,001 \\
\hline Tempo de internação (dias) & 15 & $(5-90)$ & 14 & $(6-22)$ & 19 & $(5-90)$ & 0,001 \\
\hline Mortalidade & 04 & $(5,8 \%)$ & 01 & $(1,4 \%)$ & 03 & $(4,4 \%)$ & 0,83 \\
\hline
\end{tabular}

$A B D$ - artéria brônquica direita; VJIE - veia jugular interna esquerda.

rúrgicas e 19 pacientes $(27,9 \%)$ com complicações clínicas. A mortalidade operatória foi 5,8\% (04 pacientes) e também não mostrou diferença significativa entre os grupos $(1,4 \%$ versus $4,4 \%, p=0,83$ ) (Tabela 3 ).

A sobrevida global em três anos foi $52 \%, 31 \%$ no Grupo 1 e $70 \%$ no Grupo 2 ( $p<0,001)$. A sobrevida global em cinco anos foi $37 \%$, sendo $31 \%$ no Grupo 1 e $44 \%$ no Grupo 2 ( $p<0,007)$, conforme representado nas figuras 1 e 2 .

\section{DISCUSSÃO}

Desde a década de 1940, a esofagectomia tornou-se o tratamento padrão para o câncer de esôfago. Atualmente, a sobrevida global em cinco anos está em torno de $25 \%$ e a mortalidade operatória é menor que 5\% nos centros de grande volume. Em relação à via de acesso para a esofagectomia, os dados da literatura são divergentes ${ }^{8,9}$. Recente metanálise com 7.527 pacientes demonstrou as vantagens da via TH quando há rigorosa aderência aos padrões técnicos ${ }^{14}$.

Orringer ${ }^{13}$ realizou 1.950 esofagectomias TH desde 1976, usando tubo gástrico em $97 \%$ dos casos. Houve quatro óbitos intra-operatórios $(<1 \%)$ por hemorragia mediastinal incontrolável. Entre outras complicações intraoperatórias, perfuração da cavidade pleural foi a mais comum $(75 \%)$ e menos de $2 \%$ tiveram complicações respiratórias significantes. A taxa de fistula cervical foi $13 \%$ nos primeiros 1.000 pacientes, caindo para menos de $4 \%$ após a introdução da anastomose cervical látero-lateral com

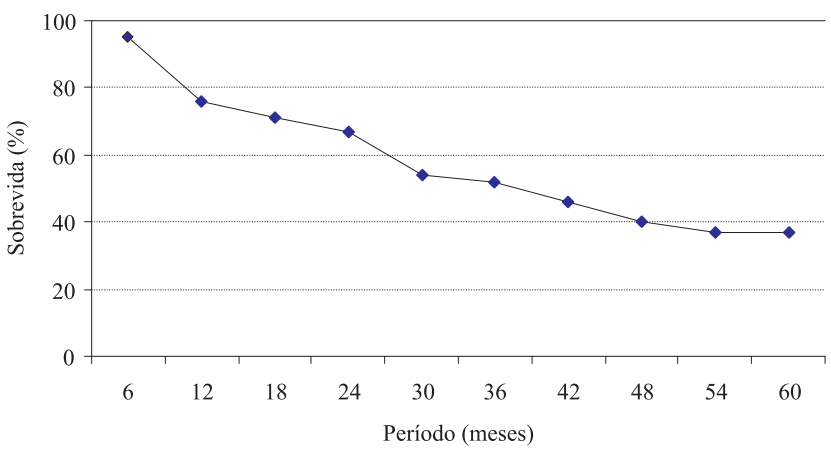

Figura 1 - Sobrevida global.

A sobrevida global em 3 e 5 anos foi $52 \%$ e 37\%, respectivamente.

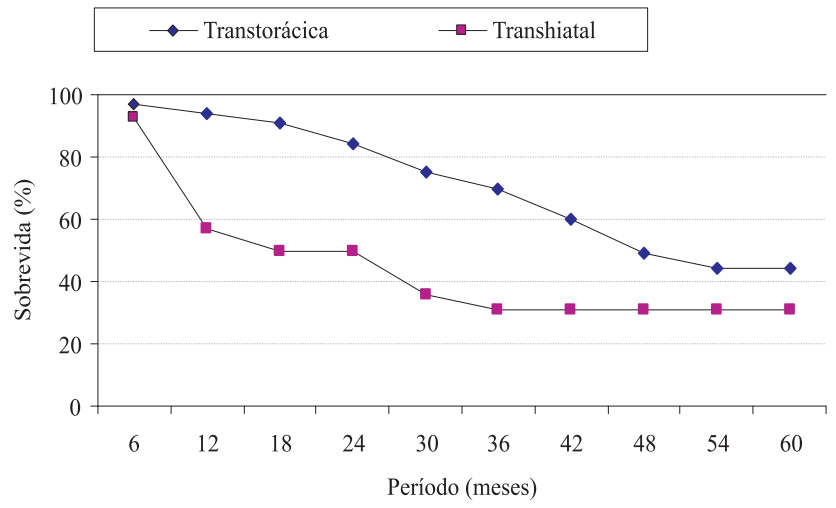

Figura 2 - Sobrevida global por grupo.

A sobrevida global em 3 anos foi 31\% no Grupo 1 versus 70\% no Grupo $2(p<0,001)$. A sobrevida global em 5 anos foi $31 \%$ no Grupo 1 versus $44 \%$ no Grupo $2(p=0,007)$. 
stappler linear de 30 milímetros, uma modificação da técnica original descrita por Collard ${ }^{15} \mathrm{em} 1998$. A necrose do tubo gástrico ocorreu em menos de $1 \%$ dos casos. A mortalidade operatória foi menor que $4 \%$ e $82 \%$ dos pacientes não tiveram complicações pós-operatórias. Para os pacientes com câncer (80\%), a sobrevida global foi $48 \%$ em dois anos e $24 \%$ em cinco anos.

Em concordância com estudos previamente realizados, ${ }^{7}$ nós encontramos maior mediana de tempo cirúrgico $(p=0,001)$ e permanência hospitalar $(p=0,001)$ no Grupo 2, fatores possivelmente contribuintes para maior morbidade cirúrgica, devido à maior exposição ao trauma cirúrgico e anestésico e a flora bacteriana nosocomial, sabidamente preditora de maior morbidade em pacientes infectados ${ }^{16,17}$. Entretanto, não houve diferença significativa neste estudo $(p=0,23)$. A mortalidade operatória global foi 5,8\% e também não mostrou diferença significativa entre os grupos $(p=0,83)$. Em uma revisão de 17.756 pacientes operados entre 1990 e 2000 a mortalidade operatória global foi $6,7 \%{ }^{18}$.

Em relação à morbidade operatória, tiveram destaque as complicações pulmonares (26,5\%) e a fístula cervical $(13,2 \%)$, ambas sem diferença significativa entre os grupos ( $p=0,67$ e $p=0,23$, respectivamente), possivelmente devido ao pequeno número de pacientes. Segundo uma metanálise ${ }^{14}$ há maior incidência de complicações pulmonares após esofagectomias por vias TT. Na série de esofagectomias trans-hiatais publicada por Orringer ${ }^{13}$ menos de $2 \%$ tiveram complicações respiratórias significantes, fato atribuído a uma seleção cuidadosa dos pacientes, além de preparo pré-operatório com abstinência de fumo por três semanas, espirometria, fisioterapia respiratória e prática de caminhadas diárias pré-operatórias, ausência de toracotomia e uso sistemático de anestesia epidural.

Recente metanálise mostra maiores taxas de fístula esofágica com a via TT ${ }^{19}$ apesar de outros grandes estudos não mostrarem diferença significativa, como o de Walther et al. ${ }^{20}$, prospectivo e randomizado, que demonstra não haver diferença na taxa de fístula entre os grupos. Em concordância com os nossos achados, a taxa de fístula anastomótica em centros de grande volume em esofagectomias está entre $8 \%$ e $13 \%$, colaborando para maior incidência de estenose esofágica ${ }^{21}$ não avaliada por nós neste estudo. Recentemente alguns autores, como Ercan et al. ${ }^{22}$ e Orringer et al..$^{23}$ têm encontrado taxas bem inferiores desta complicação (4\% e 2,7\%, respectivamente) utilizando uma modificação da técnica de sutura mecânica proposta por Colar ${ }^{15}$. Outros, como Heitmiller et al..$^{24}$ relataram taxa de fistula cervical de apenas 0,8\% em 262 pacientes consecutivos usando a técnica de anastomose manual. Em recente revisão de literatura, Mitchell ${ }^{21}$ conclui que a preferência e experiência do cirurgião provavelmente é fator mais importante que a técnica de anastomose utilizada.

Alguns estudos referem maior trauma tecidual e taxa de infecção torácica quando utilizada a via TT e mai- or risco de hemorragia e dissecção linfonodal incompleta na $\mathrm{TH}^{25,26}$. Em nosso estudo, no Grupo 1 houve um caso de hemorragia, brida, infecção de sítio cirúrgico ou infecção urinária, e no Grupo 2 houve um caso de hemorragia, empiema ou fístula pancreática.

Neste estudo predominaram os tumores do terço inferior no total, com 44 casos (64.7\%), principalmente no Grupo 1 (78,8\%), porém o Grupo 2 teve mais que o dobro de tumores do terço médio ( $48,6 \%$ versus $21,2 \%$, p $=0,02$ ), o que pode ser devido à tendência de seleção de pacientes com tumores mais proximais para a via $\mathrm{TT}$. O tipo histológico escamoso (50\%) foi mais comum que o adenocarcinoma (47\%) e o Grupo 1 teve maior incidência de adenocarcinoma $(54,6 \%$ versus $40 \%$,), porém não houve significância estatística nestes dados $(p=0,22)$. 0 grau II de diferenciação celular foi o mais comum (75\%), corroborando grandes séries da literatura ${ }^{27}$.

A média de linfonodos dissecados foi 19,1 (2 51 linfonodos), sendo significativamente maior no Grupo 2 $(21,6$ versus $17,8, p=0,04)$, porém não houve diferença significativa no número médio de linfonodos metastáticos $(4,1$ versus 3,9 linfonodos, $p=0,85)$. As margens cirúrgicas foram livres de neoplasia em todos os casos. Tal fato pode ser devido ao melhor estadiamento linfonodal obtido pela via TT, o qual sabidamente tem impacto na decisão terapêutica pós-operatória e prognóstico. Em recente estudo, Wolff et al. ${ }^{27}$ analisaram retrospectivamente 517 pacientes submetidos à esofagectomia, mostrando que mais linfonodos são ressecados pela via $T T$ em relação à $T H$ (média de 18,5 versus nove linfonodos, $p=0,001$ ), havendo diferença tanto no número de linfonodos torácicos (média 12,4 versus 4,7 linfonodos, $p=0,001$ ) como abdominais (média 6,1 versus $4,3, p=0,01$ ). Outro recente estudo de Safranek et al. ${ }^{28}$ confirma estes achados, com média de 27,5 linfonodos ressecados via TT versus 15 linfonodos via TH $(p=0,001)$.

A sobrevida global estimada em cinco anos no nosso estudo foi $37 \%$, superior a da literatura atual ${ }^{20,29}(20 \%$ a $27,9 \%$ ), provavelmente devido ao fato de termos selecionados tumores em estádios iniciais operados primariamente, sendo significativamente maior nos pacientes do Grupo $2(44 \%)$ quando comparado com o Grupo $1(31 \%)(p=$ $0,007)$. Ressaltamos que a sobrevida global em três anos foi $52 \%$, sendo mais que o dobro no Grupo 2 (70\%) em relação ao Grupo 1 (31\%) $(p<0,001)$. Podemos inferir que os pacientes do Grupo 1 tiveram recidiva mais precoce e progressão mais rápida da doença em relação ao Grupo 2. Vários estudos mostram que o número de linfonodos metastáticos não somente está associado a fatores como grau de diferenciação histológica, profundidade de invasão tumoral e tamanho tumoral, como também é fator prognóstico independente após esofagectomia ${ }^{30,31}$.

A baixa eficácia da esofagectomia em termos curativos está diretamente associada à disseminação sistêmica precoce do câncer de esôfago devido à histologia característica do órgão, com rico sistema de drenagem lin- 
fática e vascularização abundante ${ }^{32}$. Outro motivo é a relação anatômica intrínseca do esôfago que está próximo a estruturas vitais como veia cava superior, aorta e vias aéreas superiores (estruturas que podem tornar a cirurgia RO impraticável ${ }^{33,34}$. Muitos pacientes já apresentam metástases ocultas no momento do diagnóstico. Em recente estudo prospectivo, Gananadha et al. ${ }^{35}$ mostram a utilidade do FDG-PET scan (2-fluoro-2-deoxyglucose positron emission tomography) no estadiamento de pacientes candidatos à tratamento cirúrgico após realização de tomografia computadorizada e ultrassonografia endoscópica quando indicada. Houve contra-indicação cirúrgica em 25\% dos pacientes devido à disseminação nodal ou orgânica à distância detectada pelo FDG-PET e confirmada por biópsia.

Devido à baixa efetividade da ressecção cirúrgica isolada, outras modalidades terapêuticas têm sido desenvolvidas, geralmente em combinação com a ressecção. Estas associações envolvem radioterapia, quimioterapia ou tratamento combinado, podendo ser neoadjuvante e/ou adjuvante. A radioquimioterapia neoadjuvante pode obter taxas de resposta completa até $25 \%$, apesar de estudos recentes relatarem maior morbimortalidade operatória ${ }^{36-}$ 38. Em recente ensaio multicêntrico randomizado ${ }^{39}$, a quimioterapia neoadjuvante isolada mostrou aumento de $9 \%$ na sobrevida global em relação a cirurgia primária. Existe ainda a opção de tratamento combinado com quimiorradioterapia radical exclusiva (sem ressecção) ${ }^{40,41}$ hoje aceita como tratamento primário das lesões esofágicas localmente avançadas (T3 e T4) e nos pacientes com contra-indicação clínica ao tratamento cirurgico ${ }^{42} \mathrm{com}$ relatos de sobrevida média de 12 a 18 meses e global de $15 \%$ em cinco anos ${ }^{43,44}$. Na falha locorregional com tal terapia (até $60 \%$ em algumas séries), pode-se realizar a esofagectomia de resgate em casos selecionados, que cursa com alta morbimortalidade quando comparada à esofagectomia primária ou após neoadjuvância, que aplica menor dose de irradiação e um menor intervalo de tempo entre o término do tratamento combinado e a ressecção esofágica (4 -6 semanas).

A esofagectomia por câncer de esôfago, apesar de apresentar elevada morbidade operatória, permanece como o tratamento padrão para pacientes com doença ressecável e sem contra-indicação clínica. A redução da mortalidade operatória atualmente observada decorre de uma melhor seleção de pacientes para a esofagectomia, além de maior nível de especialização da equipe cirúrgica e atenção voltada para os cuidados per e pós-operatórios, refletindo em melhor controle oncológico e prognóstico mais favorável.

Apesar de ser um estudo retrospectivo e ter uma amostra relativamente pequena, na experiência do Instituto Nacional de Câncer a esofagectomia transtorácica apresentou resultados semelhantes à trans-hiatal em termos de morbimortalidade, apesar de maior tempo cirúrgico e permanência hospitalar. A sobrevida global em três e cinco anos foi maior com a transtorácica, possivelmente decorrente da maior freqüência de estágios iniciais em pacientes submetidos à transtorácica. Portanto, os resultados deste trabalho, ao contrário de outros recentes na literatura, sugerem que a via de acesso deve ser analisada individualmente, considerando o estadiamento e condições clínicas do paciente.

\section{Agradecimentos} cer-I / INCA.

Pedro Carvalho - Estatístico do Hospital do Cân-

Márcia Cavalcante - Secretária da Seção de Cirurgia Torácica do INCA.

\title{
A B S T
}

\begin{abstract}
Objective: Analyses of morbidity, mortality and overall survival after transhiatal (TH) or transthoracic (TT) esophagectomy. Methods: Retrospective non randomized study of 68 patients with esophagus neoplasia operated in the Brazilian National Cancer Institute between 1997 and 2005. We divided in two groups: Group 1 - TH (33 patients); and Group 2 - TT (35 patients). Results: The mean age was 40,7 years old ( $25-74$ years old), being 73,5\% male. Middle third tumors predominated in Group $2(48,6 \%$ vs. 21,2\%, $p$ $=0,02)$. The mean of dissected lymph nodes was biggest in Group $2(21,6$ vs. 17,8 lymph nodes, $p=0,04)$, however without difference in number of metastatic lymph nodes (4,1 vs. 3,9 linfonodos, $p=0,85)$. The mean of operative time was higher in Group 2 (410 vs. 270 minutes, $p=0,001$ ). Also the mean of length of stay was higher in Group 2 ( 19 vs. 14 days, $p=0,001$ ). The operative morbidity was $50 \%$, without statistical difference between the groups $(42,4 \%$ vs. $57,1 \%, p=0,23)$. Esophageal leakage occurred in $13,2 \%$ of cases, also without statistical difference $(9,1 \%$ vs. $17,1 \%, p=0,23)$. The mortality was $5,8 \%$ (04 patients), without statistical difference $(1,4 \%$ vs. $4,4 \%, p=0,83)$. Conclusion: In our study, the morbidity and mortality showed no statistical difference in relation to the access performed, although higher operative time and length of stay were observed in $T T$ access. The 3 and 5-years overall survival also were biggest in TT access, probably due to the biggest frequency of patients on initial stages between the submitted to the TT access.
\end{abstract}

Key words: Esophageal neoplasms. Esophagectomy. Morbidity. Mortality.

\section{REFERÊNCIAS}

1. Altorki N. En-bloc esophagectomy: the three-field dissection. Surg Clin North Am. 2005; 85(3): 611-9.
2. BRASIL. Ministério da Saúde. Secretaria de atenção a Saúde. Instituto Nacional de Câncer. Coordenação de Prevenção e Vigilância do Câncer. Estimativas 2008, Incidência de Câncer no Brasil. Rio de Janeiro: INCA, 2007. 
3. Enzinger PC, Mayer RJ. Esophageal cancer. N Engl J Med. 2003; 349(23): 2241-52.

4. Kleinberg LR, Forastiere AA, Heitmiller RF. Cancer of the esophagus. In: Abeloff MD, Armitage JO, Niederhuber JE, Kasten MB, McKenna WG, editors. Clinical oncology. $3^{\text {rd }}$ ed. Philadelphia: Churchill Livingstone; 2004. p. 1787-818.

5. Brown LM, Devesa SS, Fraumeni JF. Epidemiology of esophageal cancer. In: Posner M, Vokes EE, Weichselbaum RR, editors. Cancer of the upper gastrointestinal tract. Hamilton: BC Decker; 2002. p. 1.

6. Lerut T, Coosemans W, Decker G, de Leyn P, Ectors N Fieuws S et al; Leuven Collaborative Workgroup for Esophageal Carcinoma. Extracapsular lymph node involvement is a negative prognostic factor in T3 adenocarcinoma of the distal esophagus and gastroesophageal junction. J Thorac Cardiovasc Surg. 2003; 126(4): 1121-8.

7. Márton S, Szakmány T, Papp A, Cseke L, Horváth PO. Does transtoracic compared to transhiatal resection alter the early postoperative course of oesophagectomy? Dis Esophagus. 2005: 18(3): 155-9.

8. Tilanus HW, Hop WC, Langenhorst BL, van Lanschot JJ. Esophagectomy with or without thoracotomy. Is there any difference? J Thorac Cardiovasc Surg. 1993; 105(5): 898-903.

9. Lewis I. The surgical treatment of carcinoma of the oesophagus: with special reference to a new operation for growths of the middle third. Br J Surg. 1946; 34: 18-31.

10. McKeown KC. Total three-stage oesophagectomy for cancer of the oesophagus. Br J Surg. 1976; 63(4): 259-62.

11. Orringer MB, Sloan $H$. Esophagectomy without thoracotomy. J Thorac Cardiovasc Surg. 1978; 76(5): 643-54

12. Whooley BP, Law S, Alexandrou A, Murthy SC, Wong J. Critical appraisal of the significance of intrathoracic anastomotic leakage after esophagectomy for cancer. Am J Surg. 2001; 181(3): 198203.

13. Orringer MB. Transhiatal esophagectomy without thoracotomy. Op Tech Thorac Cardiovasc Surg. 2005; 10(1):63-83

14. Hulscher JB, Tijssen JG, Obertop H, van Lanschot JJ. Transthoracic versus transhiatal resection for carcinoma of the esophagus: a meta-analysis. Ann Thorac Surg. 2001; 72(1): 306-13.

15. Collard JM, Romagnoli R, Goncette L, Otte JB, Kestens PJ. Terminalized semimechanical side-to-side suture technique for cervical esophagogastrostomy. Ann Thorac Surg. 1998; 65(3): 814-

16. Van Lanschot JJO, Hulscher JBF, Buskens CJ, Tilanus HW, Tem Kate FJW, Obertop H. Hospital volume and hospital mortality for esophagectomy. Cancer. 2001; 91(8): 1574-8.

17. Bolton JS, Sardi A, Bowen JC, Ellis JK. Transhiatal and transthoracic esophagectomy: a comparative study. J Surg Oncol. 1992; 51(4): 249-53.

18. Jamieson GG, Mathew G, Ludemann R, Wayman J, Myers JC, Devit PG. Postoperative mortality following oesophagectomy and problems in reporting its rate. Br J Surg. 2004: 91(8): 943-7.

19. Hulscher JB, Tijssen JG, Obertop H, van Lanschot JJ. Transthoracic versus transhiatal resection for carcinoma of the esophagus: a meta-analysis. Ann Thorac Surg. 2001; 72(1): 306-13.

20. Walther $B$, Johansson J, Johnsson F, Von Holstein CS, Zilling T. Cervical or thoracic anastomosis after esophageal resection and gastric tube reconstruction: a prospective randomized trial comparing sutured neck anastomosis with stapled intrathoracic anastomosis. Ann Surg. 2003; 238(6): 803-12; discussion 812-4.

21. Mitchell JD. Anastomotic leak after esophagectomy. Thorac Surg Clin. 2006; 16(1): 1-9.

22. Ercan S, Rice TW, Murthy SC, Rybicki LA, Blackstone EH. Does esophagogastric anastomotic technique influence the outcome of patients with esophageal cancer? J Thorac Cardiovasc Surg. 2005; 129(3): 623-31.

23. Orringer $M B$, Marshall $B$, lannettoni MD. Eliminating the cervical esophagogastric anastomotic leak with a side-to-side stapled anastomosis. J Thorac Cardiovasc Surg. 2000; 119(2): 277-88.
24. Heitmiller RF, Fischer A, Liddicoat JR. Cervical esophagogastric anastomosis: results following esophagectomy for carcinoma. Dis Esophagus. 1999; 12(4): 264-9

25. Briel JW, Tamhankar AP, Hagen JA, DeMeester SR, Johansson J, Choustoulakis E et al. Prevalence and risk factors for ischemia, leak, and stricture of esophageal anastomosis: gastric pull-up versus colon interposition. J Am Coll Surg. 2004; 198(4): 536-41; discussion 541-2.

26. Hulscher JB, van Sandick JW, de Boer AG, Wijnhoven BP, Tijssen $J G$, Fockens $P$ et al. Extended transthoracic resection compared with limited transhiatal resection for adenocarcinoma of the esophagus. N Eng J Med. 2002; 347(21): 1662-9.

27. van Sandick JW, Obertop H, Fockens P, Kesecioglu J, Overdijk LE, van Lanschot JJ. [Transhiatal esophagus resection without thoracotomy for carcinoma: complications, hospital mortality and prognosis in 115 patients]. Ned Tijdschr Geneeskd. 2000; 144(43): 2061-6.

28. Safranek PM, Sujendran V, Baron R, Warner N, Blesing C, Maynard ND. Oxford experience with neoadjuvant chemotherapy and surgical resection for esophageal adenocarcinomas and squamous cell tumors. Dis Esophagus. 2008; 21(3): 201-6.

29. Zhang X, Watsin DI, Jamieson GG, Lally C, Bessell JR, Devitt PG. Outcome of oesophagectomy for adenocarcinoma of the oesophagus and oesophagogastric junction. ANZ J Surg. 2005; 75(7): 513-9

30. Eloubeidi MA, Desmond $R$, Arguedas MR, Reed CE, Wilcox CM. Prognostic factors for the survival of patients with esophageal carcinoma in the U.S.: the importance of tumor length and lymph node status. Cancer. 2002; 95(7): 1434-43.

31. Tachibana M, Yoshimura H, Kinugasa S, Dhar DK, Shibakita M, Ohno $S$ et al. Clinicopathologic factors correlated with number of metastatic lymph nodes in oesophageal cancer. Dig Liver Dis.2001; 33(7): 534-8.

32. Burmeister BH, Smithers BM, Gebski V, Fitzgerald L, Simes RJ, Devitt $P$ et al. Trans-Tasman Radiation Oncology Group; Australian Gastro-Intestinal Trial Group. Surgery alone versus chemoradiotherapy followed by surgery for ressectable cancer of the esophagus: a randomised controlled phase III trial. Lancet Oncol. 2005; 6(9): 659-68.

33. Swisher SG, Deford L, Merriman KW, Walsh GL, Smythe R, Vaporicyan A et al. Effect of operative volume on morbidity, mortality, and hospital use after esophagectomy for cancer. J Thorac Cardiovasc Surg. 2000; 119(6):1126-32.

34. Patel AN, Buenaventura PO. Current staging of esophageal carcinoma. Surg Clin North Am. 2005; 85(3): 555-67.

35. Gananadha S, Hazebroek EJ, Leibman S, Berry H, Osgood L, Shon $\mathrm{IH}$ et al. The utility of FDG-PET in the preoperative staging of esophageal cancer. Dis Esophagus. 2008; 21(5): 389-94.

36. MacGuill M, Mulligan E, Ravi N, Rowley S, Byrne PJ, Hollywood D et al. Clinicopathologic factors predicting complete pathological response to neoadjuvant chemoradiotherapy in esophageal cancer. Dis Esophagus. 2006; 19(4): 273-6.

37. Berger AC, Scott WJ, Freedman G, Konski A, Weiner L, Cheng JD, Goldberg M. Morbidity and mortality are not increased after induction chemoradiotherapy followed by esophagectomy in patients with esophageal cancer. Semin Oncol. 2005; 32(6 Suppl): S16-20.

38. Reynolds JV, Ravi N, Hollywood D, Kennedy MJ, Rowley S, Ryan A et al. Neoadjuvant chemoradiation may increase the risk of respiratory complications and sepsis after transthoracic esophagectomy. J Thorac Cardiovasc Surg. 2006; 132(3): 54955.

39. Medical Research Council Oesophageal Cancer Working Group. Surgical resection with or without preoperative chemotherapy in oesophageal cancer: a randomised controlled trial. Lancet. 2002; 359(9319): 1727-33

40. Nishimaki T, Shimoji $H$, Sunagawa $H$. Recent changes and the future roles of esophageal cancer surgery. Ann Thorac Cardiovasc Surg. 2004; 10(6): 324-32. 
41. Das P, Fukami N, Ajani JA. Combined modality therapy of localized gastric and esophageal cancers. J Natl Compr Canc Netw. 2006; 4(4): 375-82.

42. Mooney MM. Neoadjuvant and adjuvant chemotherapy for esophageal adenocarcinoma. J Surg Oncol. 2005; 92(3): 230-8.

43. Rebecca W, Richard M. Combined chemotherapy and radiotherapy (without surgery) compared with radiotherapy alone in localized carcinoma of the esophagus. Cochrane Database Syst Rev 2001; 2: D002092.

44. Minsky BD. Carcinoma of the esophagus. Part 1: Primary therapy. Oncology. 1999; 13(9): 1223-36.
Recebido em 18/02/2009

Aceito para publicação em 20/04/2009

Conflito de interesse: nenhum

Fonte de financiamento: nenhuma

\section{Como citar este artigo:}

Prisco P, Pinto CE, Barros AV, Reis J, Almeida HIB, Linhares E. Esofagectomia trans-hiatal versus transtorácica: experiência do INCa. Rev Col Bras Cir. [periódico na Internet] 2010; 37(3). Disponível em URL: http://www.scielo.br/rcbc

Endereço para correspondência:

Carlos Eduardo Pinto

E-mail: caudu2003@yahoo.com.br 